6. Conner RL, Koo K-E, Landry JR. 1974. Isovaleric acid as a precursor of odd numbered iso fatty acids in Tetrahymena. Lipids 9, 554-9.

7. Dewey VC. 1967. Lipid composition, nutrition and metabolism, in Kidder GW, ed., Chemical Zoology (Protozoa), Academic Press, New York, 1, 161-274.

8. - - Kidder GW. 1953. Factors affecting the requirement of Tetrahymena pyriformis (geleii) for folic acid. J. Gen. Microbiol. 9, 445-53.

9. — 1953. Nature of the keto acids accumulated in thioctic acid deficiency. Fed. Proc. 12, 196.

10. - 1964. Compounds sparing the requirement for biopterin. Fed. Proc. 23, 376.

11. _- 1966. Effects of long-chain mono- and dicarboxylic acids on the pteridine requirement of Crithidia. Arch. Biochem. Biophys. 115, 401-6.

12. - - Parks RE Jr, Kidder GW. 1950. Growth responses of Tetrahymena geleit to changes in the basal medium. Arch. Biochem. 29, 281-90.

13. Erwin J, Bloch K. 1963. Lipid metabolism of ciliated protozoa. J. Biol. Chem. 238, 1618-24.

14. Everhart LP, Ronkin RR. 1966. Changes in the lipids from aging populations of Tetrahymena pyriformis. J. Protozool. $13,646-50$.

15. Ferguson KA, Conner RL, Mallory FB, Mallory CW. 1972. $\alpha$-Hydroxy fatty acids in sphingolipids of Tetrahymena. Biochim. Biophys. Acta 270, 111-6.

16. Friedberg SJ, Heifetz A. 1973. Hydrogen exchange in the synthesis of glyceryl ether and in the formation of dihydroxyacetone in Tetrahymena pyriformis. Biochemistry 12, 1100-6.

17. - C, Greene RC. 1971. Loss of hydrogen from dihydroxyacetone phosphate during glyceryl ether synthesis. J. Biol. Chem. 246, 5822-7.

18. Kandatsu M, Horiguchi M. 1962. Occurrence of ciliatine (2-aminoethylphosphonic acid) in Tetrahymena. Agr. Biol. Chem. (Japan) 26, 721-2.

19. Keenan RW. 1972. Sphingolipid base phosphorylation by cell-free preparations from Tetrahymena pyriformis. Biochim. Biophys. Acta 270, 383-96.

20. Kidder GW, Dewey VC. 1951. Biochemistry of ciliates in pure culture, in Lwoff A, ed., Biochemistry and Physiology of Protozoa, Academic Press, New York, 1, 324-400.

21 _ _ 1963. Relationship between pyrimicline and lipid biosynthesis and unconjugated pteridines. Biochem. Biophys. Res. Commun. 12, 280-3.

22. 1968. A new pteridine from Tetrahymena. J. Biol, Chem. 243, 826-33.

23. - 1975. Some aspects of the initiation of protein synthesis in Crithidia fasciculata and Tetrahymena pyriformis. Comp. Biochem. Physiol. 52B, 537-9.

24. — - _ - Heinrich MR. 1954. The effects of non-ionic detergents on the growth of Tetrahymena. Exp. Cell Res. 7, 256-64.

25. - - - Rembold H. 1967. The origin of unconjugated pteridines in Crithidia fasciculata. Arch. Mikrobiol. $\mathbf{5 9}, 180-4$.

26. Mallory FB, Gordon JT, Conner RL. 1963. The isolation of a pentacyclic triterpenoid from a protozoan. $J . A m$. Chem. Soc. 85, 1362-3.

27. Nathan HA, Cowperthwaite J. 1955. "Crithidia factor" - a new member of the folic acid group of vitamins. $J$. Protozool. 2, 37-42.

28. Snell EE, DiMari SJ, Brady RN. 1970. Biosynthesis of sphingosine and dihydrosphingosine by cell-free systems from Hansenula cifferi. Chem. Phys. Lipids 15, 116-38.

29. Stoffel W, Bauer E, Stahl J. 1974. The metabolism of sphingosine bases in Tetrahymena pyriformis. Sphingosine kinase and sphingosine-l-phosphate lyase. Hoppe-Seyler's Z. Physiol. Chem. 355, 61-74.

30. Taketomi T. 1961. Phospholipids in Tetrahymena pyriformis W. Z. Allg. Mikrobiol. 1, 331-40.

31. Thompson GA Jr. 1967. Studies of membrane formation in Tetrahymena pyriformis. I. Rate of phospholipid biosynthesis. Biochemistry 6, 2015-22,

\title{
Effect of Acetate on Esterase C Activity During the Growth Cycle of Paramecium*
}

\author{
SALLY LYMAN ALLEN and THOMAS A. NERAD $\dagger$ \\ Division of Biological Sciences, The University of Michigan, \\ Ann Arbor, Michigan 48109
}

\begin{abstract}
SYNOPSIS. Enhanced esterase $\mathrm{C}$ activity could be demonstrated by starch gel electrophoresis in various stocks of Paramecium spp. ( $P$. primaurelia stocks 90 and $540, P$. biaurelia stock $93, P$. tetraurelia stock $29, P$. pentaurelia stock $87, P$. octaurelia stocks 31 and 300, and $P$. multimicronucleatum species 3, stock $8 \mathrm{MO}$ ) grown in Adaptation Medium. This esterase, however, was barely detectable when they were cultivated in Axenic Medium. Addition of trypticase to Adaptation Medium resulted in reduction of esterase $\mathrm{C}$ in the ciliates. This effect is ascribable to $\mathrm{Na}$ acetate present in trypticase. Since esterase $\mathrm{C}$ increased with the decrease in acetate concentration (as estimated by gas-liquid chromatography) during growth of Paramecium, acetate appears to be utilized by the cells. Sensitivity of esterase $\mathbf{C}$ to acetate occurs in all 6 species of Paramecium examined. Different stocks reithin a species may have different levels of sensitivity; in one case this is genetically determined. The results emphasize the importance of controlling and manipulating growth conditions for the assessment of inter- and intraspecies variations in the isozymes of Paramecium.
\end{abstract}

Index Key Words: Paramecium primaurelia stocks 90 and $540 ; P$. biaurelia stock $93 ; P$. tetraurelia stock $29 ; P$. pentaurelia stock $87 ; P$. octaurelia stocks 31 and $300 ; P$. multimicronucleatum, species 3 , stock $8 \mathrm{MO}$; esterase $\mathrm{C}$; inhibition by acetate; Adaptation Medium; Axenic Medium; starch gel electrophoresis; gas liquid chromatography.

$\mathrm{T}$ HE pattern of isozymes revealed by gel electrophoresis is commonly used as a means for assessing the degree of variation within and between species and can be translated into a measure

* This investigation was supported by Research Grant GM 15879 , from the National Institute of General Medical Sciences, U.S. Public Health Service.

† Current address: American Type Culture Collection, 12301 Parklawn Drive, Rockville MD 20852. of evolutionary distance. The variations observed in gels are limited to differences in mobility, heat stability, or activity. Activity differences may range from complete absence of an enzyme to slight differences, which may, or may not, be determined by genetic factors. The effect of a null allele may be mimicked by an environmental effect which inhibits the expression of an isozyme. In species comparisons where genetic data cannot be obtained it therefore becomes important to determine whether an environmental effect exists, and, if present, to characterize it. 


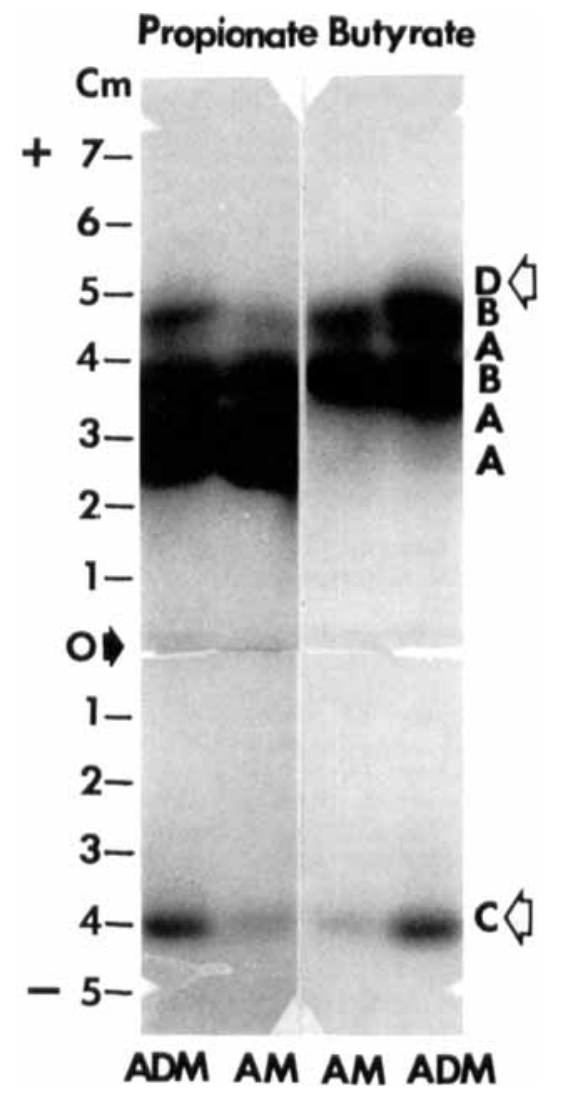

Fig. 1. Comparison of the esterases of $P$. tetraurelia, stock 29, grown in Adaptation Medium (ADM) and Axenic Medium (AM). The esterases are identified in terms of their class (A,B.D, or D). All classes are demonstrated using $\alpha$-naphthyl propionate as substrate, but only the B.C and D esterases appear with $\alpha$-naphthyl butyrate as substrate. The esterases of particular interest (cathodal $\mathrm{C}$ and anodal $\mathrm{D}$ ) are indicated by the open arrows. Distances in migration are marked off in centimeters from the origin $(\mathrm{O})$ on the left margin (Figs. 1-6).

For some time we have been concerned with assessing the intra- and interspecies variation in the isozymes of the 14 species of the aurelia complex of Paramecium $(1,5-7,9,10)$. Recently we extended ou analysis to include $t$ (or more) other species complexes. All specie's of Paramecium normally feed on bacteria. Since the presence of living bacteria not only complicates observations on isozymes, but bacterial products can be detected (5, $13,18)$, the ciliates are transferred to axenic culure for enzyme analysis. In devising a method for the simultaneous establishment of many axenic cultures, we found that media which contained autoclaved bacteria supported vigorous and indefinite growth of all stocks examined from 22 different species of Paramerium (8). Indefinite growth, however, is not supported in a medium lacking autoclaved bacteria. This difference suggests that the autoclaved hacteria contribute additional growth factors. Are there also differences in the expression of enymes of Paramecium grown in different media?

It was evident from examination of the estcrases that there are indeed differences in expression. In paramecia there are t different classes of esterases (A, B. C. and D) which are characterized on the basis of substrate specificity and sensitivity to the inhibitor eserine. Of particular interest is a difference in the expression of a $C$ esterase in paramecia grown in different media. This esterase was shown earlier to be "present" in most species

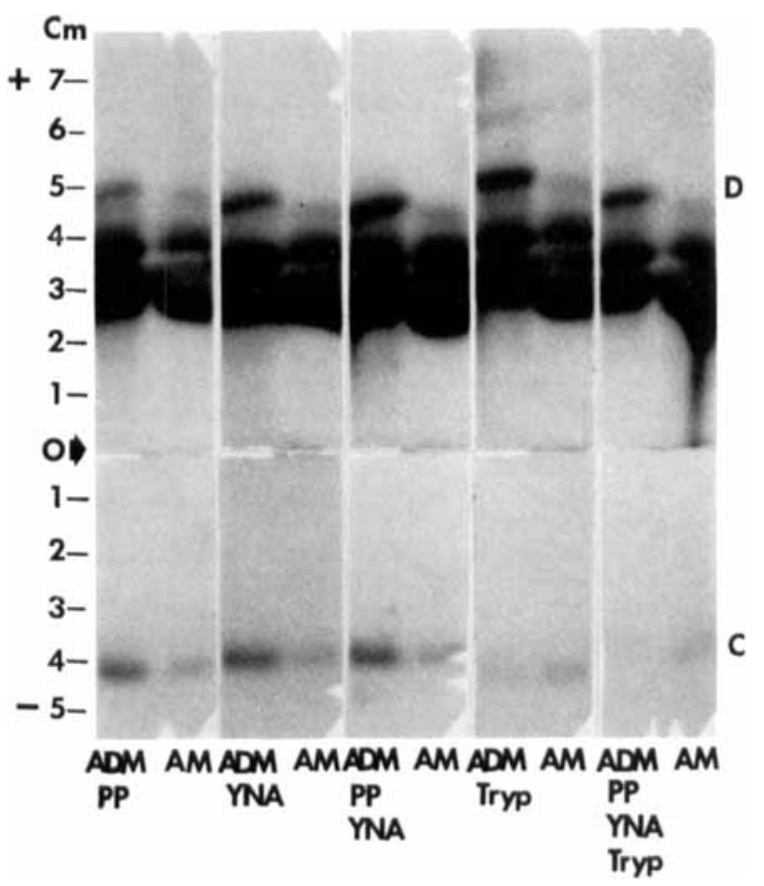

Fig. 2. Comparison of the $\mathrm{C}$ (and D) esterases of P.tetraurelia, stock 29. grown in Axenic Medium (AM) and Adaptation Medium (ADM) with the addition of components from Axenic Medium. PP, proteose peptone; YNA, yeast nucleic acid; Tryp, trypticase. The substrate is $\alpha$-naphthyl propionate.

of the aurclia complex but either "absent" or "weak in activity" in other species. In addition a difference in activity between stocks of $P$. primaurelia was shown to be due to a single gene difference (11). In this paper we show that this esterase is sensitive to a particular factor present in the growth medium of Soldo et al. (19).

\section{MATERIAIS AND METHODS}

Slocks

Axenically grown stocks derived from 6 species of Paramecium were used: P. primaurelia stocks 90 and 540, P. biaurelia stock 93, $P$. tetraurclia stock $29, P$. pentaurelia stock $87, P$. octaurelia stocks 31 and 300 , and $P$. multimicronucleatum, species 3 stock $8 \mathrm{MO}$. Species names were introduced recently by Sonneborn for each of the species in the $P$. aurelia complex (22) thereby supplanting the numbered species designation introduced previously (21) and the older designation, syngen (20).

\section{Culture Media}

Arenic Medium.--The individual components of this medium, protcose-peptone, trypticase, $\mathrm{MgSO}_{4}$, yeast nuclcic acid, TEM-4T (tartaric acid esters of beef tallow monoglycerides), stigmasterol, and vitamins, were added at the final concentrations specified by Soldo et al. (19). The method of preparation of this medium was described previously (8).

Maintenance Medium.-This medium consists of the same components as Axenic Medium, and in the same final concentrations; however, it also contains autoclaved bacteria (final concentration: $100 \mu \mathrm{g}$ dry weight $/ \mathrm{ml}$; usually Enterobacter aerogenes unless otherwise specified).

Adaptation Medium.-We described this medium in a previous report (8). It contains Peter's salts solution, autoclaved bacteria 


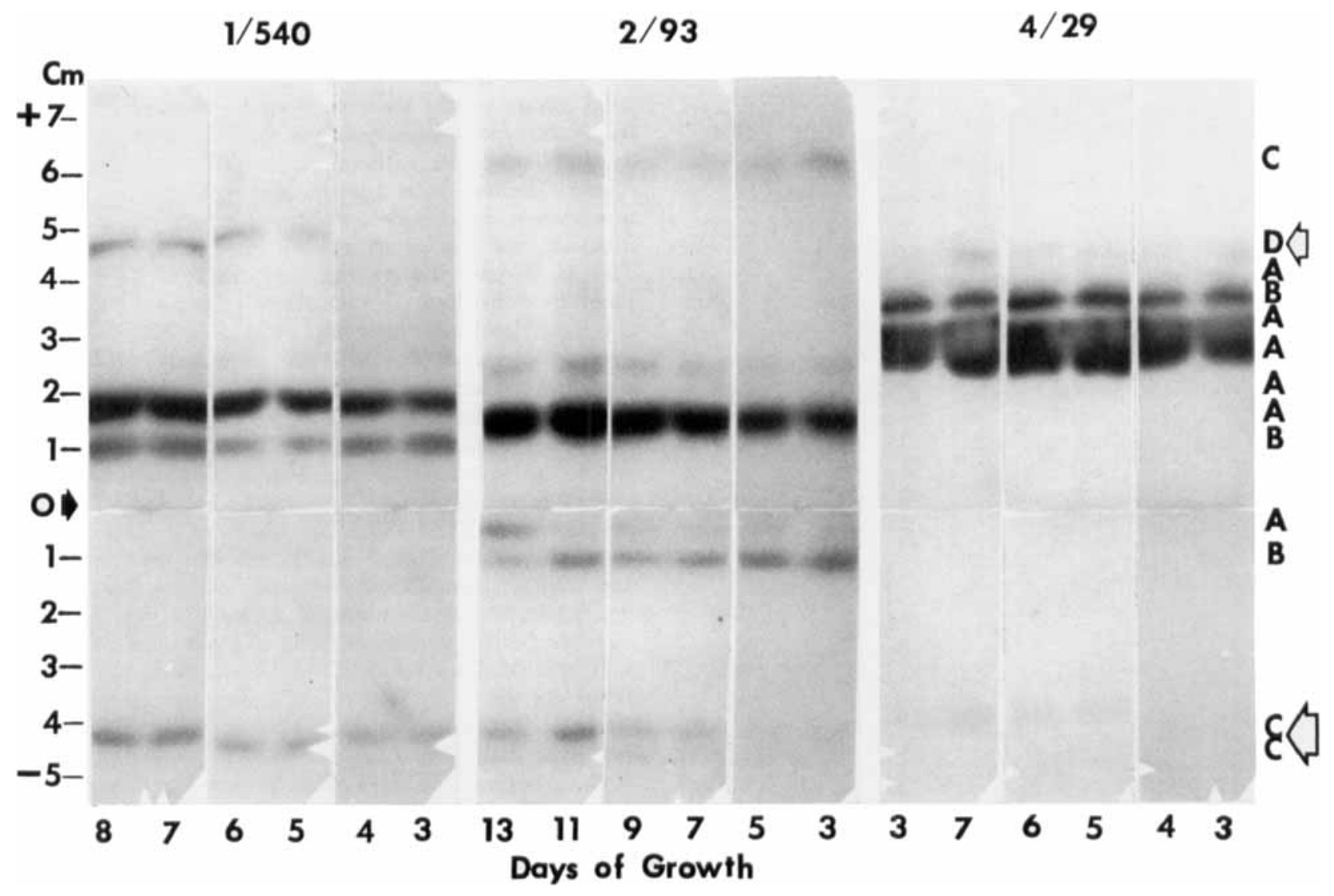

Fig. 3. Comparison of the $\mathrm{C}$ (and D) esterases during the growth cycle in Axenic Medium in P. primaurelia stock 540, $P$. biaurelia stock 93 and $P$. tetraurelia stock 29. The esterases of the stocks are identified as to class on the right of the photograph, the particular $\mathrm{C}$ and $D$ esterases of interest being indicated by the open arrows. The substrate is $\alpha$-naphthyl propionate. The days of growth of each sample are indicated below the gels.

(final concentration, $100 \mu \mathrm{g}$ dry weight $/ \mathrm{ml}$, and usually $E$. aerogenes unless otherwise specified), and stigmasterol and vitamins at the same final concentration as in Axenic Medium.

\section{Biochemical Analyses}

Identification of Esterases.-For enzyme extracts, all cultures were grown at $23 \mathrm{C}$ and harvested by centrifugation; extracts of the cell concentrates were made by freeze-thawing. Electrophoresis of whole-cell extracts was carried out in $12 \%$ starch gels using Electrostarch, as described previously (5, 7, 9, 10). Two substrates were used routinely: $\alpha$-naphthyl propionate and $\alpha$ naphthyl butyrate. All esterases are observed in a reaction mixture which includes $\alpha$-naphthyl propionate, while one class is missing (the A esterases) in the reaction mixture containing $\alpha$ naphthyl butyrate. Details of the histochemical procedures may be found elsewhere (5).

Gas-Liquid Chromatography.-Quantitative analyses of the acetate concentrations in the medium were performed using a Packard Gas Chromatograph, Model 7401, equipped with a flame ionization detector (Packard Instrument Company, Inc., Downers Grove, Illinois). The samples to be analyzed were prepared by passing $5 \mathrm{ml}$ of a Paramecium culture through a $0.45 \mu$ Milliporc filter. Two-ml of each filtrate were then acidified with $0.3 \mathrm{ml}$ of $3 \%(\mathrm{w} / \mathrm{v})$ metaphosphoric acid and frozen. On the day of analysis the samples were thawed and centrifuged at $12,000 \mathrm{rpm}$ in a microfuge (Microfuge 152, Beckman Instruments, Southfield, Michigan) for $10 \mathrm{~min}$. Three- $\mu \mathrm{l}$ aliquots of the supernatant fluid were injected into the gas chromatograph. Nitrogen served as the carrier gas at a flow rate of $30 \mathrm{ml} / \mathrm{min}$. The hydrogen flow rate was maintained at $30 \mathrm{ml} / \mathrm{min}$ and the oxygen flow rate at 400 $\mathrm{ml} / \mathrm{min}$. The inlet and detector temperatures were $145 \mathrm{C}$, while the column temperature was maintained at $135 \mathrm{C}$. The volatile fatty acid column was prepared by coating $1.4 \mathrm{~g}$ of FFAP (Varian Aerograph, Walnut Creek, California) on $10 \mathrm{~g}$ of Chromosorb W (60/80 mesh; Johns Manville Co., obtained from Anspec Co., Inc., Ann Arbor, Michigan). The column was conditioned overnight at $180 \mathrm{C}$. The concentration of acetic acid in the sample was computed relative to standards with known concentrations of this compound, according to the method developed by Dr. David P. Chynoweth, School of Public Health, University of Michigan.

\section{RESULTS AND CONCLLSIONS}

Effect of the Medium on the Expression of the Esterases.Flasks containing a liter of Adaptation Medium were inoculated with $100 \mathrm{ml}$ of a 4-day flask-culture of $P$. tetraurelia stock 29, growing in Adaptation Medium, and were grown for $t$ days. Flasks containing a liter of Axenic Medium were inoculated with $100 \mathrm{ml}$ of a 7-day flask-culture of stock 29, growing in Axenic Medium, and were grown for 7 days. Extracts were prepared, starch gel electrophoresis was carried out, and the gels were incubated in the appropriate histochemical reagents for the esterases. The esterases observed for the 2 substrates, $\alpha$-naphthyl propionate and $\alpha$-naphthyl butyrate are compared in Fig. 1. The patterns are similar except for 2 esterases - a cathodally 
(a)

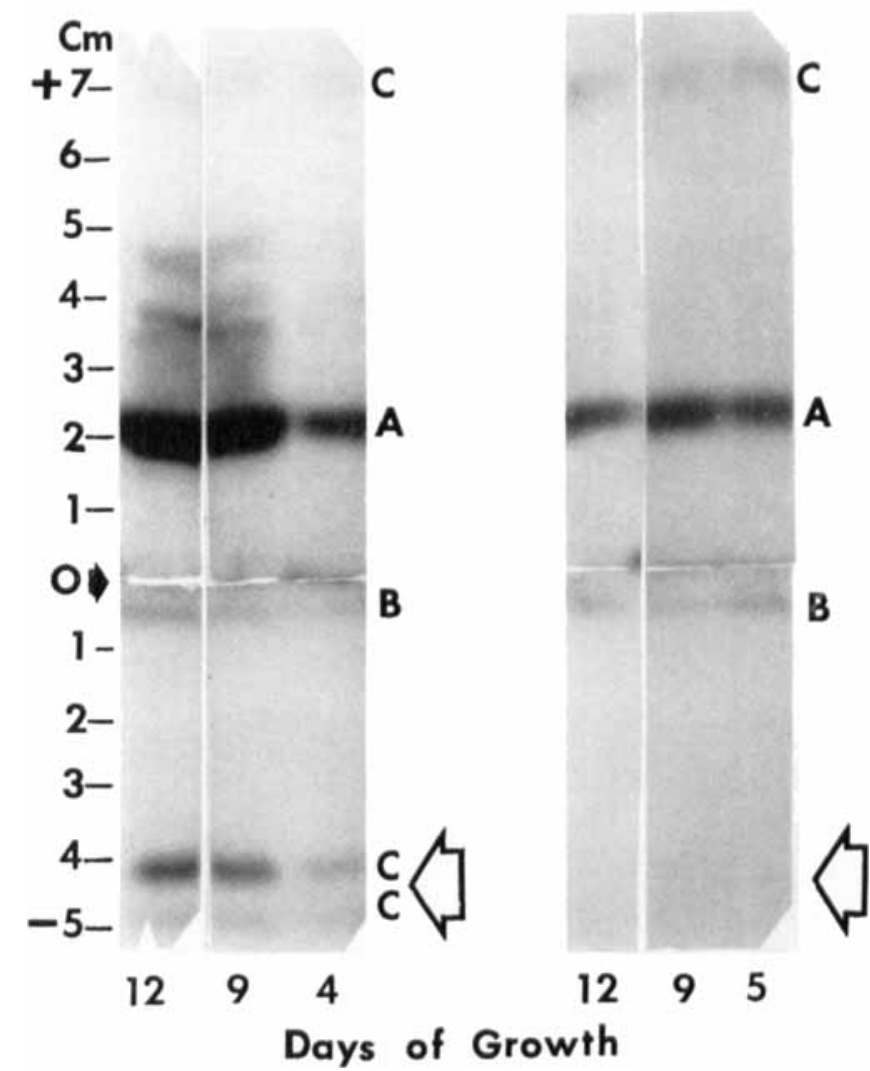

Fig. 4. Esterases during the growth cycle of $P$. biaurelia. stock 93. grown in modified Axenic Media. (a) enhancement of esterase C when trypticase is removed; (b) disappearance of esterase $C$ when $10 \mathrm{~mm}$ sodium acetate is substituted for trypticase. Open arrows indicate regions of interest. The days of growth of cach sample are indicated below the gels. The substrate is $\alpha$-naphthyl propionate.

migrating $C$ esterase, present in both mediat but much more intense in Adaptation Medium, and a rapidly migrating $D$ esterase, barely detectable in Axenic Medium and very intense in Adaptation Medium.

Effect of the Type of lipids on the Differcuce in Expression of the Esterases.-To investigate whether substitution of bacterial lipids for TEM-4T tartaric acid esters of lyeef tallow monoglycerides), a component of Axenic Medium, might affect the expression of the esterases, $t$ different bacterial species, the Gram-positive, Staphlococcus aureus and Sireptococcus focalis, and the Cram-negative Enterobacter acrogencs, and Salmonella typhimurium, were used in the following experiments. The bacteria were grown in Tryptic Soy Both to the mid-log stage of growth. The cells were then washed $2 \times$ by centrifugation in sterile Peters sals solution. and the final pellet was resuspended in peters salss solution and autoclaved at $15 \mathrm{lbs}, 121 \mathrm{C}$ for $15 \mathrm{~min}$. An autoclaved suspension was then added to a liter of Adaptation Medimo to give a final concentation of 100$) \mu \mathrm{g}$ dry weight ml. A similar amount of bacterial suspension was also added to a liter of Axenic Medium minus TEM-HT. As controls we cmployed complete. Axenic Medium and Maintenance Medium. Experimental and control media were then inoculated with $100 \mathrm{ml}$ of a culture of P. totratrelia stock 29 . growing in the same medium. Cultures containing autoclawed bacteria were harvested when the media were observed to bo clear the sus- pended bacteria were removed). Cultures in Axenic Medium were harvested after 7 days of growth.

When the esterases of stock 29 growing in the various media were compared, the patterns observed were very similar regardless of the type of bacterium present or the type of medium, with 2 exceptions. The cathodal $C$ esterase was almost absent in Axenic Medium with autoclaved bacteria minus TEM-4T. It was present in complete Axenic Medium or in Maintenance Medium, but was much more active in Adaptation Medium. The D esterase, in contrast, appeared to be very active in Adaptation Medium and in Axenic Medium minus TEM-4T, but much less active in complete Axenic Medium or in Maintenance Medium. The behavior of these esterases is not correlated in the same way with the presence or absence of TEM-4T. For the D esterase, low activity is correlated with the presence of TEM-4T, suggesting that some component(s) of TEM-4T affects the expression of this esterase. For the $\mathrm{C}$ esterase, low activity is correlated with the presence of Arenic Medium (and the absence of TEM-4T). This observation suggested to us that some other component of Axenic Medium not found in Adaptaiion Medium (protense-peptone, trypticase, or yeast nucleic acid) was responsible for the observed decrease of this esterase activity. The fact that the activity of esterase $C$ was faint or not detectable in Axenic Medium minus TEM-4T suggested that more of this factor was left at the time of harvesting than in complete Axenic Medimm. This inference semed logical since a lower population density of Paramecium was observed at plateau phase in Axenic Medium without TF.M-4T than in complete Axenic Medium.

Effect of the components of Axenic Medium on Esterase $C$ Actirity.-To determine if proteosc-peptone, yeast nucleic acid, ar trypticase, present in Axcnic, but not in Adaptation Medium, were responsible for reduction of the cathodal C esterase activity, these components were added to the latter medium individually or in combination (proteose-peptone + yeast nucleic acid or proteose-peptone + yeast nucleic acid + trypticase). In addition to the 5 variously supplemented batches of Adaptation Medium, a batch of Axenic Medium was employed. Each of these batches was inoculated with $P$. tetraurelia stock 29, growing in the same medium. The cultures grown on batches of the supplemented Adaptation Medium were harvested when the medium was cleared of bacteria; that grown in Axenic Medium was harvested on postinoculation day 7 .

The esterase patterns are shown in Fig. 2. Note that when protense-peptone, or yeast nucleic acid, or proteose-peptone and yeast nucleic acid were added, the activity of esterase $C$ was high and similar in activity to Adaptation Medium without any additions). Additions of trypticase, or trypticase in combination with proteose-peptone and ycast nucleic acid, resulted in reduction of esterase $C$ activity, less activity being observed with the combination of components. This observation suggests that a factor found in trypticase, but also present in small amounts in other components of the modium, causes reduction in esterase $\mathrm{C}$ activity.

Does the Pattern of Esterasc $C$ Activity change during the Crouth Cycl' - In this experiment 2 different stocks of Paramecium P. primaurelia stock 540 and P. tetraurelia stock 29) each growing in Adaptation and Axenic Medium wore harvested after increasing numbers of days of growth. In addition, samples of $P$. biaurelia stock 93, growing in Axenic Medium, wcre examined after different postinorulation days. When stocks 540 and 29 were grown in Adaptation Medium, the activity of esterase $\mathrm{C}$ remained high throughout the growth cycle. As shown in Fig. 3, however, when the 3 stocks were rultivated in Axenic Medium, esterase $C$ increased in activity as the cultures aged. This pattern of expression might be expected if the factor in trypticase respon- 


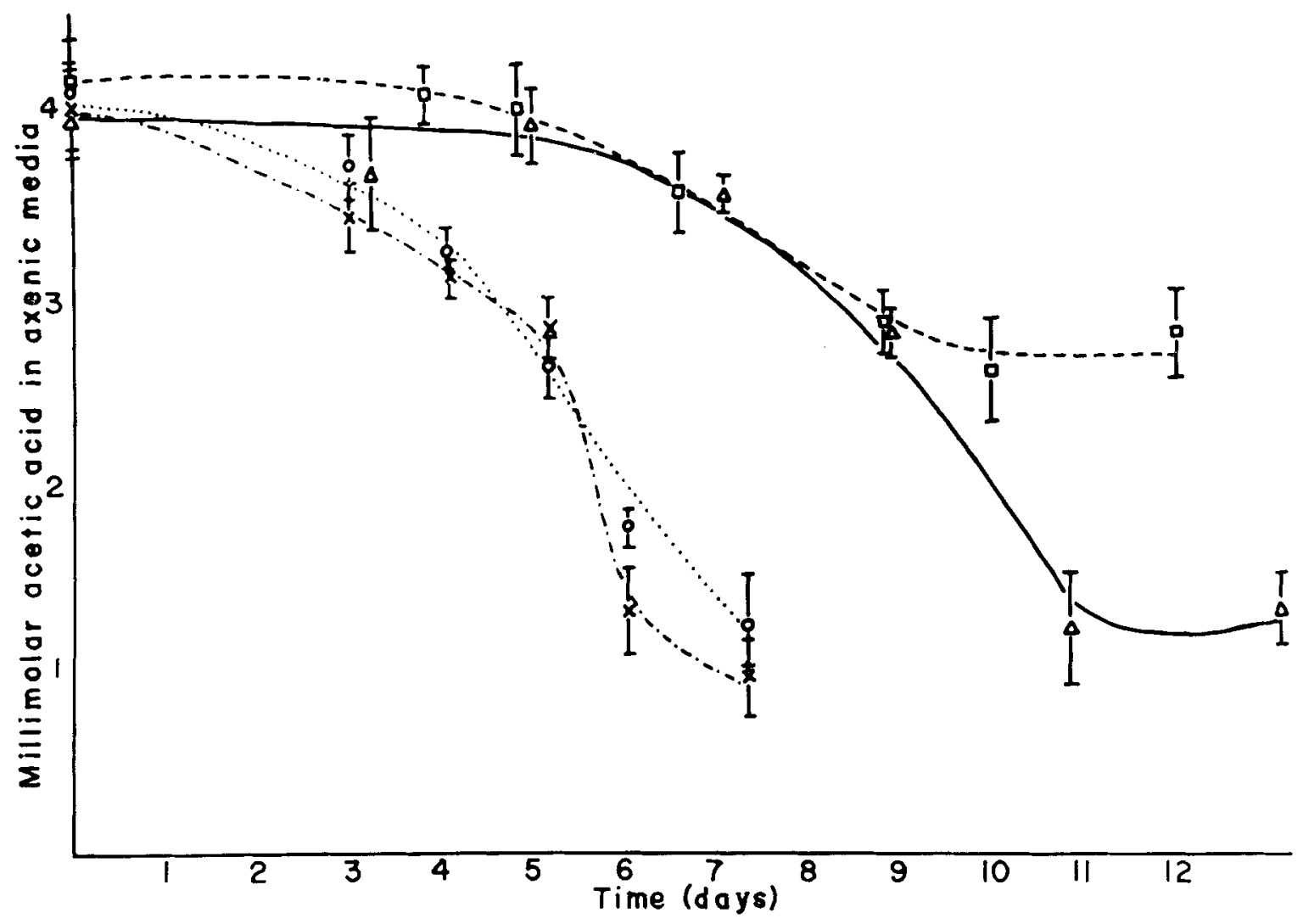

Fig. 5. Acetic acid concentration in Axenic Medium during the growth cycle of $P$. primaurelia stock 90 ( $\square---\square$ ); $P$. primaurelia stock $540(X-\cdot-\times) ; P$. biaurelia stock $93(\triangle-\triangle) ; P$. tetraurelia stock $29\left(O_{-}-\bigcirc\right)$. For each sample the mean measurement is given, the bar indicating $95 \%$ confidence limits.

sible for reduced esterase $\mathrm{C}$ activity, and present in Axenic but not in Adaptation Medium, is slowly being used up during the growth cycle.

Is the component affecting Esterase $C$ Activity used up during growth?-If esterase $C$ activity is enhanced because a factor present in trypticase is used up during growth, we might expect that without trypticase in Axenic Medium esterase $\mathrm{C}$ should be active throughout the growth cycle. This possibility was tested with $P$. biaurelia stock 93, grown in Axenic Medium without trypticase. Samples, harvested on 4,9 and 12 postinoculation days, were examined for their esterase activities (Fig. 4a). After 4 days of growth in this medium esterase $\mathrm{C}$ was as active as in extracts taken from 7-9 day cultures growing in complete Axenic Medium. By 9 days the intensity of the band was greater than that observed at any stage in the growth cycle in the latter medium.

A clue as to the identity of the factor responsible for reduced esterase $\mathrm{G}$ activity came from the statement made by van Wagtendonk (23) that a mixture composed of $\mathrm{Na}$ acetate, $\mathrm{CaCl}_{2}$ and the trace elements $\mathrm{Fe}, \mathrm{Zn}, \mathrm{Mn}$ and $\mathrm{Cu}$ could be substituted for trypticase in Axenic Medium. We tested this by replacing trypticase with $10 \mathrm{~mm} \mathrm{Na}$ acetate and growing $P$. biaurelia stock 93 in this medium for 5,9 and 12 days. Esterase $C$ was not detected in any of the samples taken throughout the growth cycle (Fig. $4 \mathrm{~b})$. From these experiments we conclude that the factor in trypticase to which esterase $\mathrm{C}$ is sensitive is $\mathrm{Na}$ acetate and that this compound is used up during the growth cycle. It was evident from measurements of the amount of acctate in the various components of Axenic Medium by gas-liquid chromatography that $93 \%$ of the acetate is contributed by the trypticase com- ponent. This distribution correlates with the previous finding that most, but not all, of the observed reduction in esterase $\mathrm{C}$ activity could be attributed to the presence of trypticase.

Do other Species of Paramecium have an Acetate-sensitive Esterase $C$ ? - In the 1 st experiment, 2 different species of Paramecium and 2 different concentrations of acetate were used. Paramecium primaurelia stock 540, and P. tetraurclia stock 29 were grown in Adaptation Medium, Adaptation Medium with $1 \mathrm{~mm} \mathrm{Na}$ acetate or in Adaptation Medium with $10 \mathrm{~mm} \mathrm{Na}$ acetate. In the presence of $1 \mathrm{~mm} \mathrm{Na}$ acetate the activities of the cathodal $\mathrm{C}$ esterases were very weak; they were totally absent in the presence of $10 \mathrm{~mm} \mathrm{Na}$ acetate. In the 2nd experiment $P$. biaurelia stock $93, P$. pentaurelia stock $87, P$. octaurelia stocks 31 and 300 , and $P$. multimicronucleatum, species 3 , stock $8 \mathrm{MO}$ were grown in Adaptation Medium with and without $10 \mathrm{~mm} \mathrm{Na}$ acetate. Whenever acetate was present, the cathodal $\mathrm{C}$ esterases were not detected. These stocks have $\mathrm{C}$ esterases of different mobilities; thus, despite the differences in mobility (and the presumed genetic alteration which changes mobility), all these enzymes are sensitive to acetate. We conclude that acetate sensitivity is a general response of esterase $\mathrm{C}$ in species of Paramecium.

Does the Acetate Concentration deline with Groteth in Axenic Medium?-Paramecium primaurelia stocks 90 and 540, P. biaurelia stock 93, and $P$. tetraurelia stock 29 were cultivated in Axenic Medium and harvested at different stages of growth. For each sample, the concentration of acetate in the culture was determined by gas-liquid chromatography and the esterase patterns by starch gel electrophoresis. The results of the acetate 

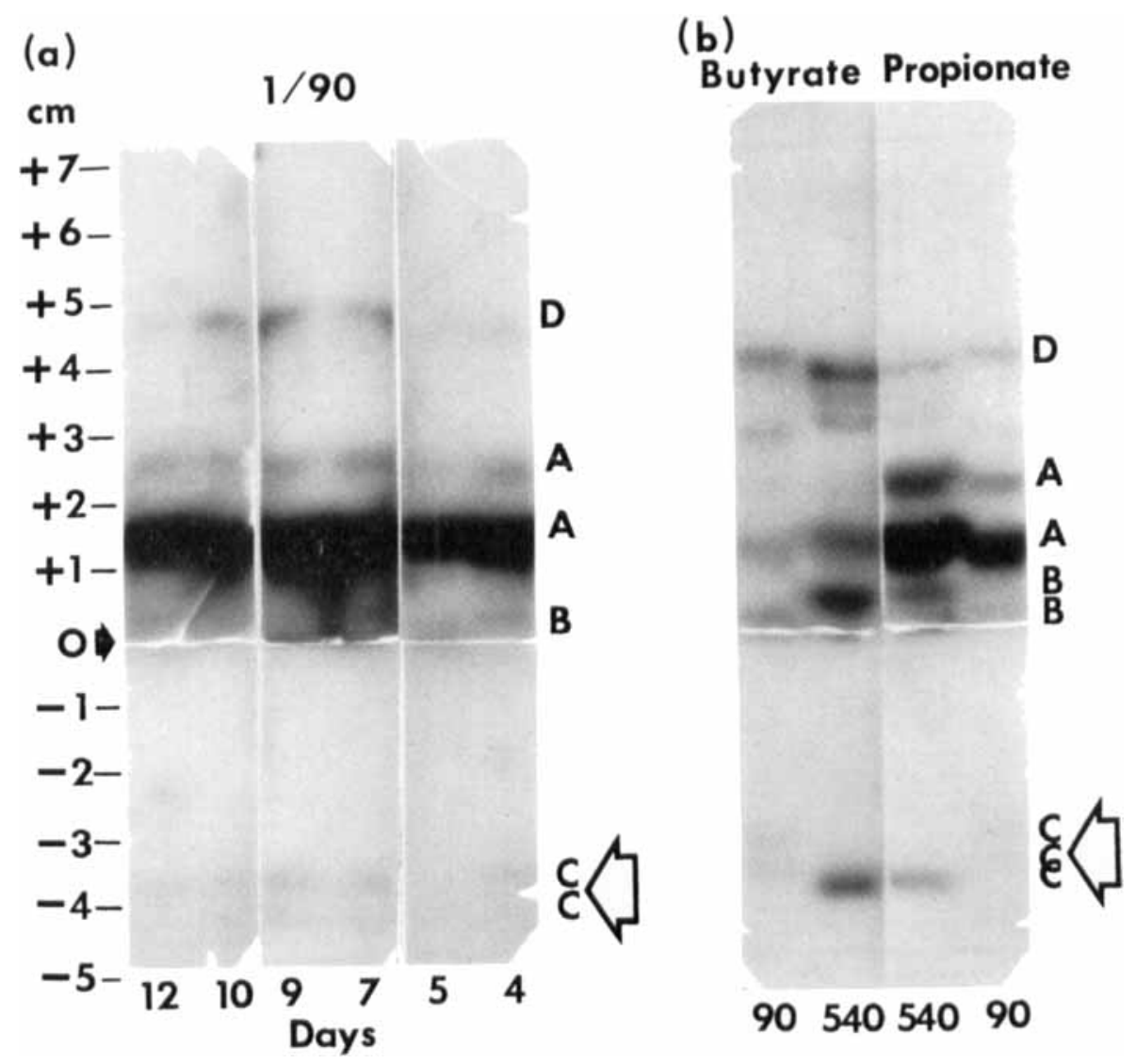

Fig. 6. Esterase C activity in stock 90 of $P$. primaurelia (a) during the growth cycle in Axenic Medium; (b) compared to stock 540 of $P$. primaurelia in Adaptation Medium. The esterases of the stocks are identified as to rlass on the right of the photographs. The $C$ esterases of interest are indicated by the open arrows. In (a) the days of growth of each sample are indicated below the gels, and the substrate is $\alpha$-naphthyl propionate. In (b) the results with both $\alpha$-naphthyl propionate and $\alpha$-naphthyl butyrate are shown.

determinations are shown in Fig. 5 ; those of the gel analysis are shown in Figs. 3 and 6.

In general, we conclude that as the acetate concentration is reduced the activity of the $C$ esterases increases. Differences among stocks, however, are observed. They depend, in part, on differences in growth rate and final population density, and on inherent differences in sensitivity to acetate. Stocks 29 and 540 grow more rapidly and reach higher densities than do stocks 90 and 93. Thus, there is a temporal delay in the acetate concentration decrease in the latter 2 stocks, and this delay is accompanied by a delay in the appearance of the $C$ esterases. Innate differences in sensitivity atso ocrur, the (? esterase in stock 540 being more active carly than in other stocks. Of particular interest is the difference in esterase $C$ activity between stocks 90 and 540 (Figs. 3. 6), both members of the same species, $P$. frimaurelia, in which a single gene has been found to control this difference 11 ). In old cultures of stock (10) the $\mathrm{C}$ esterase appears as a faint doublet (Fig. 6a). In Adaptation Medium, it also appears as a faint doublet, and we can see that it differs from the allozyme in stock 540 in mobility as well as in activity (Fig. 6b).

\section{I)ISCLSSION}

The cathodally migrating $C$ esterase is widespread in various species of Paramecium 5,10 . It can catalye $\alpha$-naphthyl acetate, although very ponly compared to its ability to catalyze $\alpha$-naphthyl propionate or $\alpha$-naphthyl butyrate. It is insensitive to high concentrations of eserine sulfate $(5)$. We can now add one nore property of this enzyne: during growth of Paramecium it is sensitive to acctate; therefore, its expression reflects the level of acetate present in the medium. Axenic Medium contains acctate primarily in trypticase. As acetate is consumed during the growth cycle of Paramecium, the acetate concentration in the medium decreases and the esterase $\mathrm{C}$ activity increases. We do not know what the real function of this enzyme is in the cell, nor do we know how it is affected by acetate.

Genetic factors as well as the conditions of growth determine the pattern of esterases observed after gel electrophoresis. All 4 classes of esterases are present in all species of Paramecium so far examined (Allen \& Nerad, in preparation). Single gene differences have been found for mobility differences of the $\mathrm{A}$ and $B$ esterases between stocks of the same species $(7,11)$. Variations among storks in the thermal stability of A or B esterases with similar mobility have been observed, implying further genetic differences (6). Differences in the activity of certain A estcrases in $P$. biaurclia and $P$. octaurelia have also been noted $(7,11)$, and in the case of $P$. octaurelia they appear to be genetically controlled. The cathodal $\mathrm{C}$ esterase also varies in expression which is both genetically (11) and environmentally determined, through its sensitivity to acctate.

The conditions of growth appear to be important for expression of certain esterases (7). We know that the presence of living bacteria can alter the activity of particular esterases as well as contribute new esterases $(5,13,18)$. The presence of particular components in the growth medium also influences the activity of particular $C$ and $D$ esterases, as shown in this paper. In both 
cases enhancement of activity is due to the removal of a factor present in Axenic Medium. For the $\mathrm{C}$ esterase, the factor is acetate present chiefly in trypticase. For the D esterase, a factor is present in TEM-4T (with some effect exerted also by acetate). The observed expression of these esterases depends upon when the cultures growing in Axenic Medium are examined during the growth cycle. "Old" cultures have higher esterase C activity because the concentration of acetate in the medium has been reduced.

The effect of growth conditions on the expression of enzymes is complex and not unique to Paramecium. In Tetrahymena thermophila (the new name for syngen 1 of $T$. pyriformis; see Ref. 15) the activities of individual esterase isozymes are sensitive to growth cycle changes $(2,3)$. Some of the acid phosphatases in this species also respond to growth conditions (4). In other eukaryotic microorganisms, such Aspergillus, Chlamydomonas, or Neurospora, a complex system of genetic and environmental factors determines the activities of particular phosphatases $(12,14$, $16,17)$. We do not know the manner of interaction between genetic and environmental factors in Paramecium for any of the enzymes which respond to growth conditions, i.e. whether "synthesis" or "activity" is responsible for the changes in band intensity in gels.

The fact that some of the esterases respond to growth conditions and that in some cases they change in expression with the growth cycle is important information for assessing intra- and interspecies variability. It means that in screening large numbers of stocks we must control very carefully the conditions of growth for each stock. Where we know that a particular enzyme is sensitive to particular growth conditions, as in the case of esterase $\mathrm{C}$, we can use this information to identify homologous enzymes in different species.

\section{REFERENCES}

1. Adams JP, Allen SL. 1975. Genetic polymorphism and differentiation in Paramecium, in Markert CL, ed., Isozymes IV. Genetics and Evolution, Academic Press, New York, pp. 867-82.

2. Allen SL. 1964. The esterase isozymes of Tetrahymena: their distribution in isolated cellular components and their behavior during the growth cycle. J. Exp. Zool. 155, 349-70.

3. - 1965. Genetic control of enzymes in Tetrahymena. Brookhaven Symp. Biol. 18, 27-51.

4. 1968. Genetic and epigenetic control of several isozymic systems in Tetrahymena. Ann. N.Y. Acad. Sci. 151, 190. 207.
5. - - Gibson I. 1971. Intersyngenic variations in the esterases of axenic stocks of Paramecium aurelia. Biochem. Genet. 5, $161-81$

6. _— 1975. Syngenic variations for enzymes of Paramecium aurelia, in Markert CL, ed., Isozymes IV. Genetics and Evolution, Academic Press. New York, pp. 883-99.

7. - Golembiewski, PA. 1972. Inheritance of esterases $A$ and $B$ in syngen 2 of Paramecium aurelia. Genetics 71, 469-75.

8. - Nerad TA. 1978. Method for the simultaneous establishment of many axenic cultures of Paramecium. J. Protozool., 25, 134-9.

9. 25, Byrne BC, Cronkite DL. 1971. Intersyngenic variations in the esterases of bacterized Paramecium aurelia. Biochem. Genet. 5, 135-50.

10. — Farrow SW, Golembiewski PA. 1973. Esterase variations between the 14 syngens of Paramecium aurelia under axenic growth. Genetics 73, 561-73.

11. Cavill A, Gibson I. 1972. Genetic determination of esterases of syngens 1 and 8 in Paramecium aurelia. Heredity 28, 31-7.

12. Dorn G. 1965. Genetic analysis of the phosphatases in Aspergillus nidulans. Genet. Res. 6, 13-26.

13. Gibson I, Cavill A. 1973. Effects of bacterial products on a Paramecium esterase. Biochem. Genet. 8, 37-64.

14. Matagne RF, Loppes R. 1975. Isolation and study of mutants lacking a depressible phosphatase in Chlamydomonas reinhardi. Genetics 80, 239-50.

15. Nanney DL, McCoy JW. 1976. Characterization of the species of the Tetrahymena pyriformis complex. J. Amer. Microsc. Soc. 95, 664-82.

16. Nyc JF. 1967. A repressible acid phosphatase in Neurospora crassa. Biochem. Biophys. Res. Commun. 27, 183-88.

17. —_ Kadner RJ, Crocken BJ. 1966. A repressible alkaline phosphatase in Neurospora crassa. J. Biol. Chem. 241, 1468-72.

18. Rowe E, Gibson I, Cavill A. 1971. The effects of growth conditions on the esterases of Paramecium aurelia. Biochem. Genet. 5. 151-9.

19. Soldo AT, Godoy GA, van Wagtendonk WJ. 1966. Growth of particle-bearing and particle-free Paramecium aurelia in axenic culture. J. Protozool. 13, 494-7.

20. Sonneborn TM. 1957. Breeding systems, reproductive methods and species problems in protozoa, in Mayr E, ed. The Species Problem, Am. Assoc. Adv. Sci. Symp., Washington, D.C. pp. 155-324.

21. 1975. Paramecium aurelia, in King RC, ed. Handbook of Genetics, Plenum Press, New York, N.Y., vol. 2, 469. 594.

22. 1975. The Paramecium aurelia complex of fourteen sibling species. Trans. Amer. Microsc. Soc. 94, 155-78.

23. Van Wagtendonk WJ. 1974. Nutrition of Paramecium, in van Wagtendonk WJ, ed., Paramecium-A Current Survey, Elsevier Sci. Pub. Co., New York, N.Y., pp. 339-76.

\section{BOOK REVIEW ...}

Starr, M. P., Ingraham, J. L. \& Balows, A., eds. 1977. Annual Review of Microbiology. Vol. 31. Annual Reviews, Inc., 4139 El Camino Way, Palo Alto, CA 94306.695 pp. $\$ 17.00$ in USA, $\$ 17.50$ elsewhere.

This book contains only 2 articles that deal with protozoa, and these are the only ones that I shall deal with. A. G. Fredrickson (University of Minnesota) wrote 25 pages with 116 references on Behavior of mixed cultures of microorganisms, and Chandler Fulton (Brandeis University) wrote 33 pages with 127 references on Cell differentiation in Naegleria gruberi.

The role of protozoa in mixed cultures is said to be primarily that of predation, mostly on bacteria. There are many unsolved problems in the predator-prey relationship, including the mitigating factors that allow both populations to co-exist. Fredrickson stresses quantitative relationships.

Cell differentiation is defined differently by almost everyone who works on it. Fulton is concerned with the ameba-flagcllate transformation of Naegleria, and especially with the events that occur during transformation. Most of his discussion deals with the formation of flagellates from amebae, but part of it deals with the reverse. This is an interesting paper about a puzzling phenomenon. What goes on here? Answer this question, and you may have a clue to the evolution process.-NORMAN D. LEVINE, College of Veterinary Medicine, Univ. of Illinois, Urbana IL 61801, USA. 